\title{
Clinical Pharmacy and Future Prospects
}

\author{
Rajesh Singla* \\ Associate Professor, Microbiology and Head, Agriculture Department, S.S.D. College of Professional Studies, Punjab, India \\ *Corresponding Author: Rajesh Singla, Associate Professor, Microbiology and Head, Agriculture Department, S.S.D. College of \\ Professional Studies, Punjab, India.
}

Received: October 18, 2019; Published: October 21, 2019

DOI: 10.31080/ASPS.2019.03.0425

Increased population in India has resulted in the shortage of Healthcare professionals. Moreover, most of the population belongs to below poverty line or either low income group and thus cannot afford the increased cost of medical aid. In India, Pharmacists do not offer any professional services like counseling, direction of medicine use, possibility of adverse drug reaction, possible drug interaction when multiple medicines are advised etc. Also, the curriculum, poor medical knowledge, lack of clinical training etc. cannot allow the pharmacists to go beyond the basic role they are offered and as such Clinical Pharmacy Practice has become the need of hour. Clinical pharmacy practices encourage the shift of single focus from product orientation to multiple engagements and maximize the benefits that a patient can obtain from the intake of medicines and as such clinical pharmacist's helps to promote healthcare facilities and prevention of disease by optimization of the medication by thereby play direct role in patient care.

There is a need to focus on pharmaceutical care based on the outcomes of use of medicines and it should be dependent on the practice of clinical pharmacy. Pharmacists and their teams must work together to help the patients to take appropriate medicines thereby improving the results and ensure their safety and avoid unnecessary treatment and reduce wastage of money and resources. With their skills and knowledge their role must be in reducing the morbidity rate during primary care in relation to medication.

Since Independence till 2010, education (curriculum) as well as pharmacist's role and practice was more oriented towards pharmaceutical industry needs viz. production, formulation, quality control and marketing rather than practicing and management in the health sector with the consequence that India lacked in the healthcare facilities. It resulted in improper treatment, ADR (adverse drug reactions), medical treatment getting out of reach of common man leading to economic loss to patients and finally misuse of drugs leading to drug abuse ultimately causing huge loss to Indian economy. During the current decade, these problems have been viewed upon and addressed with a shift of focus from traditional role of pharmacists towards clinical practice with a significant change right from the bottom with change in the curriculum, hospital based trainings, clinical practice skills etc. Profession in pharmacy is slowing shifting from pharmaceutical industry that is from technical side towards healthcare sector and would pick up in the years to come with government as well as private players having a key important role.

Government has now shifted its focus and the change is reflected in the changed curriculum in pharmacy education and also with the start of various courses with emphasis on clinical/medical practice like Doctor of Pharmacy, Postgraduate Programs in Pharmacy Practice during the current decade. The focus must be on applied therapeutics, patient data analysis, patho-physiological basis of disease, clinical pharmacokinetics as well as communication skills as per local needs. The most important part of this would be hospital based training whereby the concerned trainees would have a clear understand of all the aspects of the treatment which includes diagnostic process, lab testing and data analysis, mode and role of different treatment methods and options, effects of drugs etc. The idea seems to be very good and benefitting but is still in its infancy and it will still need a decade to flourish as mitigation of all aspects of pharmaceutical care into health system will take time as changes right from bottom which includes changes in curriculum, addition of new and upgraded courses in relation to pharmacy practice throughout the country along with changes in mode of training and clinical/hospital based training or internship, creation of posts and infrastructure with inputs from government and stake holders is required. 
In the end I would say that pharmacy as a profession has been under utilized and overlooked in the healthcare system in our country. Pharmacy practice/clinical pharmacy must be given due regard and special consideration must be given into various services in this sector and people must be made aware of the various benefits and of the rewarding career in this multi-disciplinary medical arena.

Volume 3 Issue 11 November 2019

(c) All rights are reserved by Rajesh Singla. 